\title{
Results of X-shooter data reduction software on laboratory frames
}

\author{
P. Goldoni ${ }^{a}$, F. Royer ${ }^{b}$, M. Horrobin ${ }^{c}$, P. François ${ }^{b}$, \\ L. Guglielmi $^{a}$, R. Haigron ${ }^{b}$, J. Vernet ${ }^{d}$, A. Modigliani ${ }^{d}$, P. Bristow ${ }^{d}$ \\ ${ }^{a} \mathrm{APC} / \mathrm{UMR}$ 7164, 10 rue Alice Domon et Leonie Duquet, Paris, France; \\ ${ }^{b}$ GEPI/UMR 8111, Observatoire de Paris-Meudon, 5 Place Jules Janssen, Meudon, France; \\ ${ }^{c}$ University of Amsterdam, Kruislaan 403, Amsterdam, the Netherlands; \\ ${ }^{d}$ ESO, Karl Schwarzschild Str. 2, Garching bei München, Germany
}

\begin{abstract}
We present the current state of the Data Reduction Software (DRS) being developed at APC, Paris Observatory, Amsterdam University and ESO for the X-shooter échelle spectrograph. X-shooter is the first VLT second generation instrument, which will have its first light during the fall of the current year and will be available to the astronomical community starting April 2009. The DRS will be fully integrated in the ESO VLT data flow environment and it will use the ESO Common Pipeline Library. X-shooter data have two main characteristics, on the one hand the exceptionally wide band ( $0.3-2.4$ micron) covered in a single exposure, and on the other hand the spectral format with highly curved orders and tilted lines. After a brief description of the reduction process, the main results obtained up to now on simulated and laboratory data are reported. In particular the precision of wavelength calibration and sky subtraction are discussed.
\end{abstract}

Keywords: Spectroscopy, Data Reduction, Pipeline

\section{INTRODUCTION}

\subsection{Framework of the DRS project}

$\mathrm{X}$-shooter ${ }^{1}$ is a single object spectrograph which will be installed at the Cassegrain focus of one of the UTs of the VLT in the summer of 2008. It will be the first second generation instrument to be installed at VLT. The consortium that builds X-shooter comprises the Copenhagen University Observatory (DK), INAF (Istituto Nazionale d'Astrofisica) (IT), the GEPI-Observatoire de Paris-Meudon and the Laboratoire d'Astroparticule et Cosmologie (APC)(FR), ASTRON and the Universities of Amsterdam and Nijmegen (NL) and ESO. The aggressive development schedule and the contemporary development of other second generation instruments made a large external hardware and financial contribution necessary.

The Data Reduction Software (DRS) is developed by APC, GEPI and the University of Amsterdam. The DRS is designed to be a collection of data reduction recipes based on the ESO Common Pipeline Library (CPL) operating in the VLT DFS environment. Therefore the usual Data Reduction tools of the VLT environment such as the Data Organizer, the Reduction Block Scheduler, EsoRex and Gasgano will be used in conjunction with the X-shooter pipeline. In this scheme, the consortium has developed the recipes in a modular way following templates defined at ESO which allowed to focus on instrument specific algorithms.

Further author information: (Send correspondence to P. Goldoni)

P. Goldoni: E-mail: paolo.goldoni@apc.univ-paris7.fr, Telephone: +33-1 57276912 


\subsection{Instrument overview and status}

The X-shooter is a high-efficiency spectrograph with a spectral resolution in the range 4000-10000, depending on wavelength and on slit width. X-shooter consists of a central structure (the backbone) which supports three prism-cross-dispersed échelle spectrographs optimized for the UV-Blue, Visible and Near-IR wavelength ranges respectively. The backbone contains the calibration and acquisition units, an Integral Field Unit (IFU) which can be inserted in the light path and reformats a field of $1.8^{\prime \prime} \times 4^{\prime \prime}$ into a long slit of $0^{\prime \prime} 6 \times 12^{\prime \prime}$, two dichroics to split the beam among the three arms and relay optics to feed the entrance slits of the spectrographs. The spectral format is fixed.

- X-shooter has three different arms, covering different spectral ranges:

- UVB: starting at $300 \mathrm{~nm}$. The upper limit is determined by the first dichroic which reflects the UVB light. The crossing wavelength is $558 \mathrm{~nm}$.

- VIS: starting at $558 \mathrm{~nm}$, the high pass wavelength cut being set by the first dichroic. The upper limit is determined by the second dichroic which reflects the Visual-Red light. The crossing wavelength is $1020 \mathrm{~nm}$.

- NIR: starting at $1020 \mathrm{~nm}$, the high pass wavelength cut being set by the second dichroic. The upper limit is determined by the cut off of the detector and the atmospheric absorption.

- X-shooter has two observing configurations:

- SLIT: in this case the atmospheric dispersion is compensated by ADCs in UVB and VIS arms. In NIR, the effect is not significant for $Z_{D}<60^{\circ}$.

- IFU: in the telescope focal plane the IFU pattern covers a field of $1.8^{\prime \prime} \times 4^{\prime \prime}$ and reformats it to a virtual slit image composed by three aligned slices of $00^{\prime \prime} 6$ width, $4^{\prime \prime}$ each long. All other components are as in the SLIT case. The IFU is located before the ADCs in the light path. Flux will then be lost at the entrance of the IFU in VIS, and mostly in UVB, before the light pass through the ADC.

Slits of different sizes can be inserted in the light path. The possibility to use several slits with width ranging from 0 ". 3 to $11^{\prime \prime} 5$ is currently foreseen.

The X-shooter project passed the Final Design Review in 2007, after that the consortium started building the instrument. The three X-shooter spectrographs have been built and tested in Denmark, Italy and Holland and they have been shipped to Garching. They are presently integrated and test images are routinely taken, from present results, the instrument appear to be within specifications. The Preliminary Acceptance in Europe (PAE) review will take place in July 2008 and if succesful, the instrument will be shipped to Paranal were first light is foreseen in September 2008.

\subsection{Specific Features}

The wide spectral band covered by X-shooter implies that different observing techniques will be used depending on the scientific objective of the observation and on the source intensity along the spectral band.

The key issue, as far as the NIR is concerned, is the background subtraction. The infrared sky, at wavelengths shorter than $2 \mu \mathrm{m}$, is dominated by the emission from the hydroxyl molecule $\mathrm{OH}$. This emission is highly structured, spatially and spectrally and exhibits intensity variations of up to $10 \%$ on time scales of about 5 to 15 minutes. $^{2} \quad$ At wavelengths longer than $2 \mu \mathrm{m}$, observations are generally strongly affected by thermal background radiation from the sky and the telescope, with strongly increasing background when going to longer wavelengths. Accurate sky subtraction is essential and this requires special attention and procedures. Therefore three different observing modes are available:

- staring mode: the science target is observed at a fixed position on the slit. The sky is estimated from the object frames themselves, on both sides of the object. This requires that the object is bright enough to be localized on the frame, and not too extended, to allow the estimation of the sky. This technique is the 
standard one for observations in the optical range. An accurate sky background subtraction in this mode is crucial to properly reduce data in the NIR where the sky contribution is predominant. This is described in Sect. 4.2.

- nodding mode: the classical technique in IR spectroscopy is to observe object(s) at two or more positions along the slit. These positions are usually called $\mathrm{A}$ and $\mathrm{B}$ and pairs of observations ( $\mathrm{AB}$ or $\mathrm{BA}$ ) are repeated into sequences. The $\mathrm{B}$ position is shifted with respect to $\mathrm{A}$ by an amount $\delta$ along the slit. The sky is removed at the first order by subtracting one frame from the other and thus obtaining two difference frames: A-B and B-A. The B-A frame is then shifted by $\delta$ and the resulting frame (B-A)' is summed to the $(\mathrm{A}-\mathrm{B})$ one. The extraction is then performed on the resulting frame $(\mathrm{A}-\mathrm{B})+(\mathrm{B}-\mathrm{A})$ '. This process is sometimes called double pass sky subtraction.

- on-off mode: when the object is extended (i.e. covering a large fraction of the entrance slit), the sky has to be sampled away from the object. This may result in a loss of efficiency for the observations, which can amount to $50 \%$ of the observing time if the sky has to be sampled as frequently as the object.

To each of the observing modes corresponds a dedicated science reduction recipe. The three arms will be reduced separately, using the same recipe, which will behave according to the arm. The sky emission lines present in the UVB and VIS spectral ranges can also be removed using standard nodding observation techniques. The spectra from the three arms will be merged into a single spectrum only for quick look purposes.

These three observing modes will be available in SLIT observing configuration while in IFU observing configuration only staring mode and on-off mode will be available because the IFU is too short to allow nodding in the cross-dispersion direction.

The highly curved and tilted orders require a special effort to perform wavelength calibration and optimal extraction, details are in Sect. 3 .

When the science target is a point source, and with a signal per pixel high enough to be localized and extracted, a $1 \mathrm{D}$ spectrum will be produced. In general however, the observing configurations of X-shooter, SLIT and IFU, allow the combination of spatial and spectral informations and the production of, respectively, 2D and 3D spectroscopic data. These 2D/3D products will be the default output of science reduction.

\section{DRS OVERVIEW}

The X-shooter DRS has been built to be fully integrated in the VLT-DFS pipeline making use of libraries and reduction tools developed at ESO. More precisely, the DRS reduction recipes has been written in standard ANSI C using the ESO Common Pipeline Library. The CPL is presently used by ESO as the main tool for the development of data reduction pipelines for VLT instruments.

X-shooter calibration, and especially the wavelength calibration will depend on a physical model, i.e. a numerical model of the instrument based on the engineering data. ${ }^{3}$ An optimized comparison between the physical model and the calibration data will allow to obtain the instrument configuration with much more precision than with the usual methods. The use of the physical model in wavelength calibration is detailed in Sect. 3.

\subsection{Inputs}

The DRS pipeline will receive in input the raw data to be processed and the associated calibration data:

- Raw images from the VLT instrument software

- The calibration data

Calibration data and instrument parameters are extracted from the calibration database. Two types of calibration data exist:

- master calibrations which are generated by the pipeline either by processing of daytime calibrations or attached calibrations (TBC), examples are flat fields and wavelengths solutions,

- ancillary data like standard star tables, reference line catalogs, atmospheric extinction tables, physical model parameters and other reference tables required for data reduction. 


\subsection{Outputs}

The DRS pipeline will produce the following data:

- flux and wavelength calibrated 2D (and 1D when relevant) spectra with associated errors (see Fig. 3.b and c),

- three-dimensional data cubes containing the position and the wavelength information of the observation (in case of IFU observation),

- QC parameters and performance monitoring values. The QC parameters are divided in QC0 parameters for processing control and $\mathrm{QC} 1$ parameters to check of the instrument performances with respect to the nominal ones.

Throughout all the reduction, each image will be associated with an error image and a bad pixel map. The error images and bad pixel maps will be propagated through each step. Likewise for the extracted spectra, so that the final result will be associated to a realistic estimation of the errors. The final outputs will be produced for each arm of X-shooter. A single merged spectrum from 0.3 to $2.4 \mu \mathrm{m}$ will be created for quality control purposes and a quick look of the spectrum.

\section{FIRST RESULTS OF PIPELINE ANALYSIS}

The X-shooter DRS version 0.15 has been used to extract 1-D and 2-D spectra of laboratory data. The main steps are:

- bias and dark subtraction

- background subtraction and flat field correction,

- wavelength calibration and rectification,

- object localization and extraction (see ${ }^{4}$ for details).

Presently only the first possibility is completely implemented. Here we describe the polynomial wavelength calibration procedure and we report on the results of the wavelength calibration recipes of the X-shooter DRS on VIS arm laboratory data taken in March 2008 in Garching. The analysis of the other two arms is presently ongoing and the results are similar.

\subsection{The Wavelength Calibration procedure}

In the case of X-shooter, the shape of the orders is strongly curved with a parabolic shape, and the spectral lines are tilted (see Fig. 3.a), therefore the wavelength cannot be expressed only as a function of position in the dispersion direction, but depends also on the cross-dispersion position. The position $(X, Y)$ on the detector as a function of the wavelength, order number and position on the slit $(\lambda, n, s)$ is mapped using a multi-pinhole calibration exposure (see Fig. 1). The multi-pinhole frame is illuminated by an arc lamp through a mask at the entrance slit. There are nine 0.5 holes evenly spaced along the $12^{\prime \prime}$ slit.

There are two ways to map the $(X, Y)$ space into the physical quantities $(\lambda, n, s)$. The first method, which is now fully implemented, is the polynomial solution, where $X$ and $Y$ are polynomial expressions of $\lambda, n$ and $s$. The second method is the use of a physical model of the instrument ${ }^{3}$ whose parameters can be tuned to match the observations. This second method is currently being interfaced with the pipeline, and the following results only use the polynomial wavelength solution.

The cascade for wavelength calibration involves taking the following steps:

- Using a single pinhole Th-Ar frame, a guess wavelength solution is calculated (xsh_predict)

- Using a single pinhole flat-field frame, an order table is calculated (xsh_orderpos) 


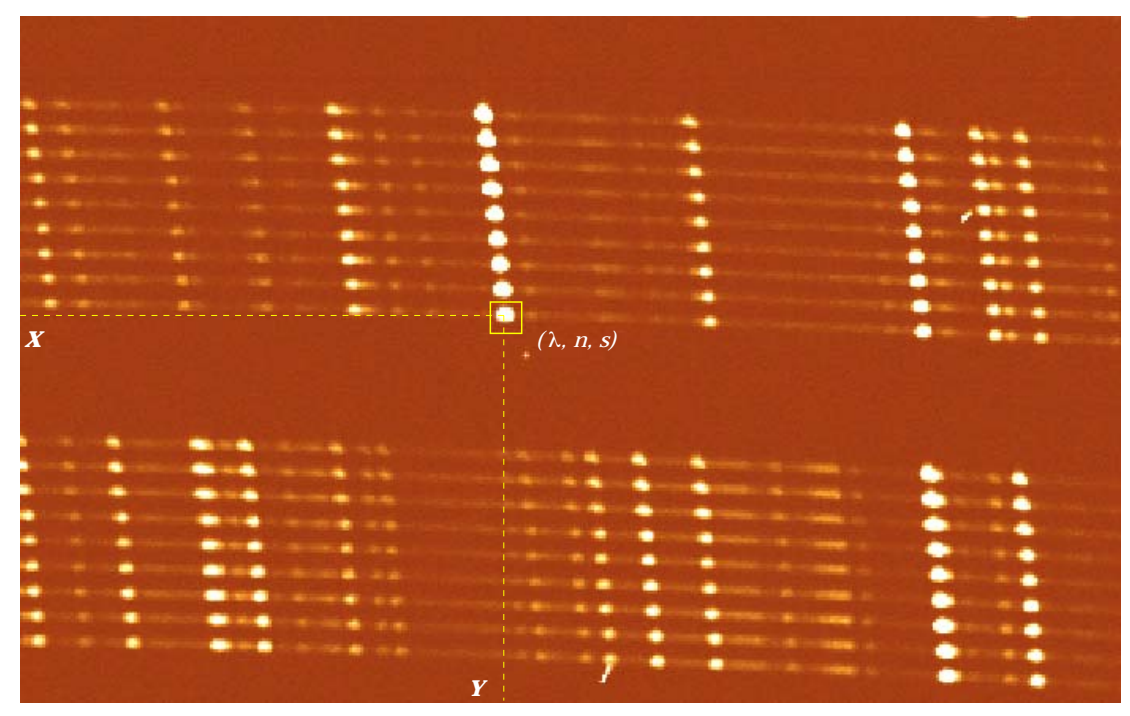

Figure 1. Example of a raw multi-pinhole calibration frame taken in March 2008. A zoomed part of the detector is shown. For a given line (in the box), with known wavelength $(\lambda)$, order number $(n)$ and position on the slit $(s)$, the position on the detector $(X, Y)$ is fitted using a 2-dimensional Gaussian.

- Using a full slit flat-field frame, the edges of the orders are calculated (xsh_mflat)

- Using a multi-pinhole Th-Ar frame, the global 2-D dispersion solution is calculated (xsh_2dmap)

The xsh_predict recipe takes as input a list of guess positions of lamp lines on the calibration frame produced by the physical model and produces a polynomial guess wavelength solution. The guess wavelength solution at this stage is valid only for the central position of the slit and is a correction of the input physical model position. The recipe xsh_orderpos localizes the orders along all their length and produces a more precise order table. This new order table is fed to the xsh_mflat recipe which, besides producing a master flat, detects the edges of the orders which give the dimension of the slit on the detector.

\subsection{The Bi-dimensional Dispersion relation}

Like the xsh_predict recipe, the mapping calibration recipe uses the physical model of the instrument to obtain a list of guess positions of the lines on the calibration frame. The physical model input is then corrected using the guess wavelength solution and the order tables produced by the previous recipes. This operation produces the actual guess positions of the lines in the multi-pinhole frame. Each of these spectral lines is then fitted by 2-dimensional Gaussians and the resulting positions $(X, Y)$ are adjusted by a global polynomial fit on the whole detector. This polynomial solution is then stored in the form of Chebyshev polynomials in the $(\lambda, n, s)$ space.

This bi-dimensional dispersion relation (spatial and spectral directions) is also used to construct a wavelength map which will be used to get the wavelength of specified pixel positions (for e.g. sky subtraction and optimal extraction), and to correct for optical distortions and resample the frames into a $(\lambda, s)$ grid for extraction of a rectified frame.

\subsection{Quality of the Global Wavelength Solution}

We were able to apply our procedure to the March 2008 laboratory frames and to obtain a global wavelength solution. In order to validate our results (pixel positions $X$ and $Y$ as a function of $\lambda, n$ and $s$ ) and estimate the quality, we processed Th-Ar frames to extract 1-D and 2-D Th-Ar and sun spectra calibrated in wavelength.

Figure 2 plots the distributions of the residuals in velocity in the VIS arm for the 0.6 slit and the $1^{\prime \prime}$. The dispersions are respectively 0.51 and $0.82 \mathrm{~km} \mathrm{~s}^{-1}$. The distributions are not centered on 0 but it may be partly 


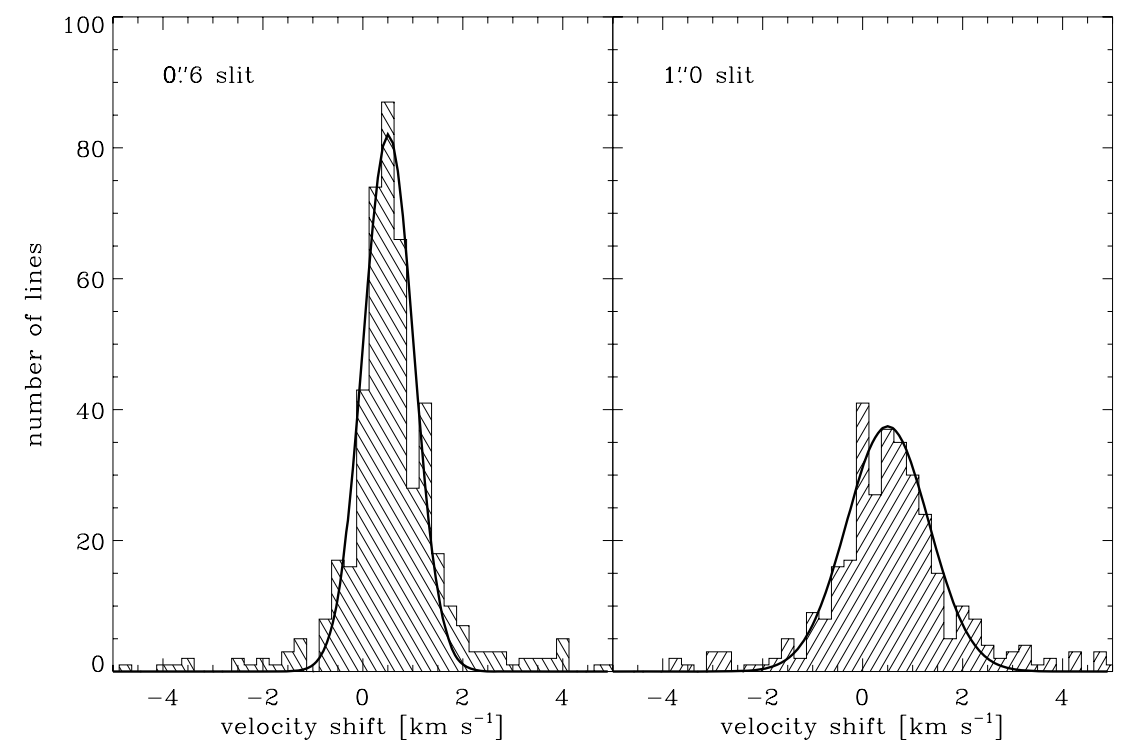

Figure 2. Distribution of velocity shifts from Th-Ar emission lines in the VIS arm. These shifts are the difference between calibrated line positions and laboratory wavelengths. The two panels correspond to two Th-Ar frames observed with respectively the $0 .{ }^{\prime \prime} 6$ slit and the $1^{\prime \prime}$ slit. They have been reduced as science frame, using the same wavelength solution, to produce 1D wavelength calibrated Th-Ar spectra. The solid thick lines are the Gaussian fit of the distributions, and their parameters are: $\mathbf{0}^{\prime \prime} \mathbf{6}$ slit: mean $=0.51 \mathrm{~km} \mathrm{~s}^{-1}$, standard deviation $=0.51 \mathrm{~km} \mathrm{~s}^{-1} ; \mathbf{1}^{\prime \prime}$ slit: mean $=0.50 \mathrm{~km} \mathrm{~s}{ }^{-1}$, standard deviation $=0.82 \mathrm{~km} \mathrm{~s}^{-1}$.

due to a systematic shift between the multi-pinhole mask (used to build the wavelength solution) and the slit. The residuals in velocity for a reduced multi-pinhole frame are centered on $0.14 \mathrm{~km} \mathrm{~s}^{-1}$.

The Sun observed through the $1^{\prime \prime}$ slit has been reduced. The wavelength solution obtained with xsh_2dmap has been used, and the 1D spectrum has been extracted on the rectified frame (Fig. 3). When compared to a solar spectral atlas*, the resulting extracted spectrum is a perfect match.

\section{ADVANCED METHODS}

\subsection{Optimal Extraction}

Optimal extraction ${ }^{5}$ is a method to extract cross-dispersed spectra delivering the maximum possible signalto-noise ratio while simultaneously preserving a good spectrophotometric accuracy. This is accomplished by adding nonuniform weights to the pixels in the extraction sum giving more weight to the pixels with more flux. The original method, ${ }^{5}$ however, is only suited for straight orders and lines and therefore it is not applicable to X-shooter. The optimal extraction function used for X-shooter will be based on the mathematical method described by Marsh ${ }^{6}$ and generalized by Piskunov and Valenti ${ }^{7}$ and by Donati. ${ }^{8}$ The approach we adopted is the one developped by Donati.

The main idea of the Marsh method is to obtain a precise determination of the cross-order and spatial profile shapes by subsampling them using several subpixels (virtual resampling). Polynomial are then used to estimate the flux of the object along the slit. This technique allows to improve the knowledge of the cross-order and spatial profiles (see Fig. 4) and therefore allow to describe the spectral shape with greater precision.

\footnotetext{
${ }^{*}$ The solar spectral atlas was obtained with the Fourier Transform Spectrometer at the McMath/Pierce Solar Telescope situated on Kitt Peak, Arizona, and operated by the National Solar Observatory, a Division of the National Optical Astronomy Observatories.
} 

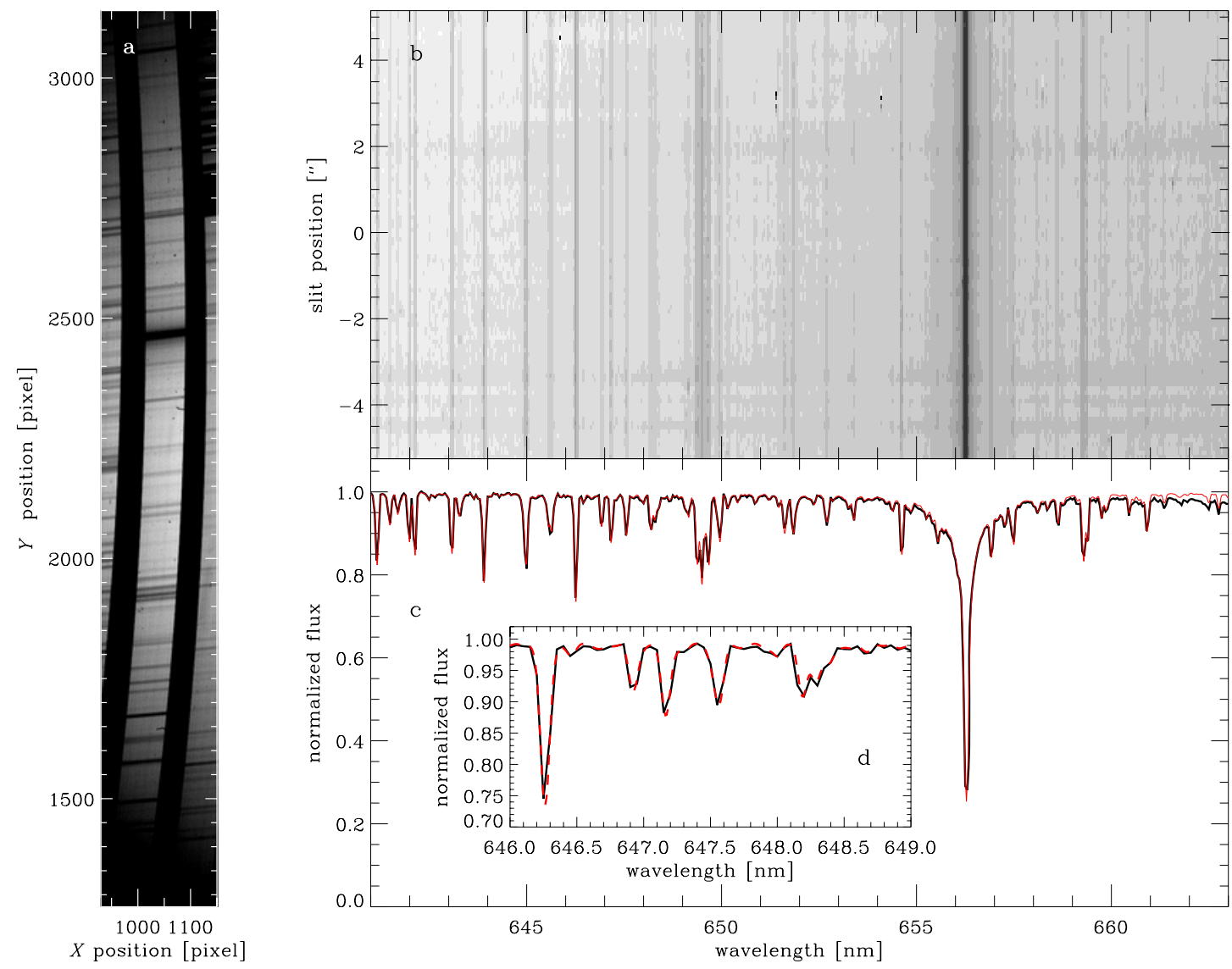

Figure 3. a. Part of a raw frame showing the échelle order \#25 in the VIS arm (containing H $\alpha$ ) of a solar spectrum taken with a full $1^{\prime \prime}$ slit exposure. The dispersion axis is along the $Y$ axis and the wavelength increases with pixel position. One can notice the order curvature and the tilt of spectral lines. b. Part of the bidimensional product reduced from a. sampled in wavelength $(0.05 \mathrm{~nm}$ step) between 641 and $663 \mathrm{~nm}$ and spatially along the slit ( 0. .' 1 step). The frame is completely rectified. c. Corresponding part of the monodimensional product, which in this case is a simple extraction on the rectified frame showed in $\mathrm{b}$. The thick solid line is the observed spectrum, which has been normalized to continuum. The gray/red solid line is a solar spectral atlas whose resolution has been degraded to match the observed one $(\sim 10000)$. d. Zoom on the observed spectrum and spectral atlas.

With the knowledge of this spatial and spectral decomposition, we can therefore apply the method of optimal extraction. At the present stage of our software development, we have assumed a gaussian cross-order profile, a type of extraction which can be necessary for extreme case where the generalized Marsh method may fail for very low $\mathrm{S} / \mathrm{N}$ spectra, as it is done for the ESO/UVES pipeline.

We applied this method to a sun pinhole observation taken at ESO labs with X-Shooter mounted on a bench. On non oversampled data, the illumination was spread over 7 pixels with more than $80 \%$ of the flux in 3 pixels. In Fig. 5 we show one of the order resampled on a curvilinear coordinate system which allows to follow the shape of the order and the variation of the tilt along the order (virtual resampling). On the left part of the figure, the estimated gaussian cross-order profile is shown. This gaussian fit is used to compute the the relative weights of the subpixels.

In Fig. 6, we show in the upper part the extracted spectrum in the region of $\mathrm{H} \alpha$ using standard extraction (i.e; equal weights) and the optimal extraction assuming a Gaussian cross-order profile. One can see that, as expected, there is no substantial improvement using optimal extraction on this kind of data where the $\mathrm{S} / \mathrm{N}$ is 


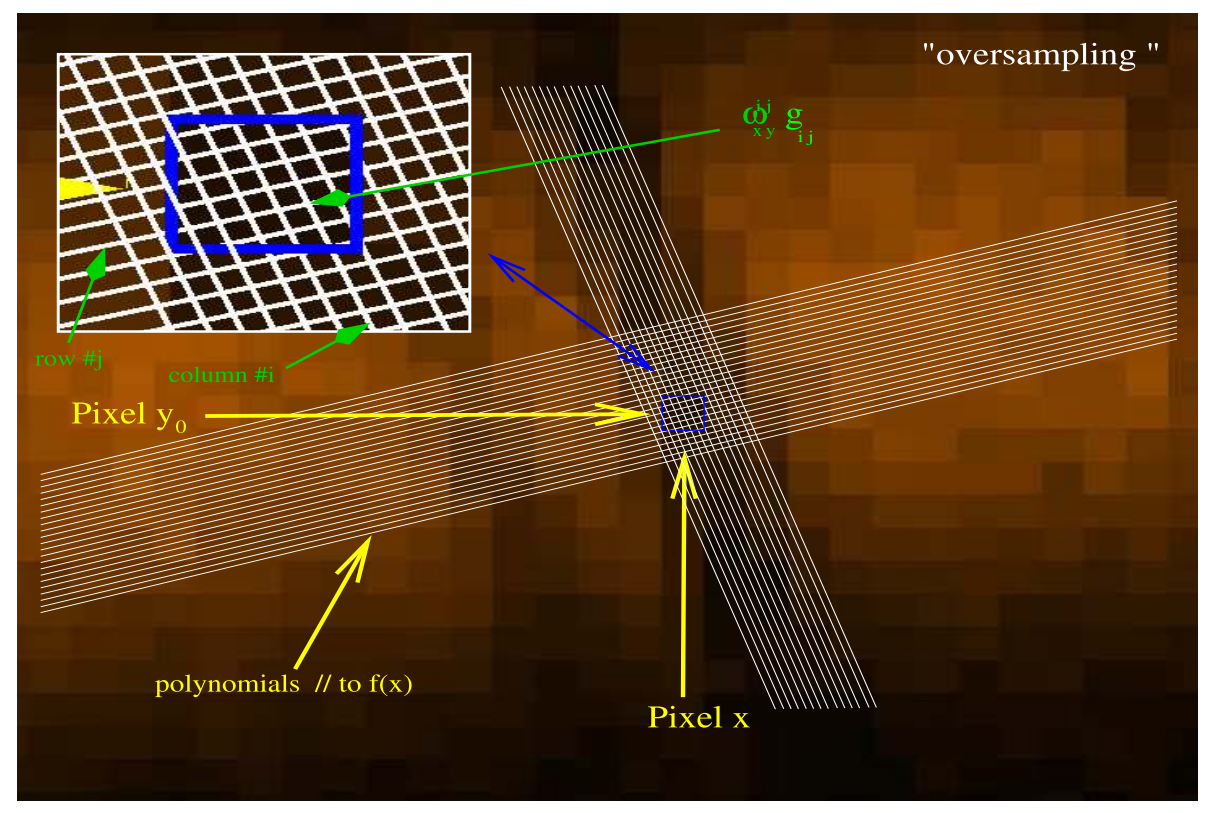

Figure 4. Description of the oversampling method. The inclined horizontal lines represent the order (the spectrum) while the inclined vertical lines represent the line (the slit). In the upper left corner we show a zoom over the pixel $\left(x, y_{0}\right)$ which is subsampled in several subpixels. The grid inside the detector pixel $\left(x, y_{0}\right)$ defines the locations where the weights $\omega_{x y}^{i j}$ have to be computed for that pixel.

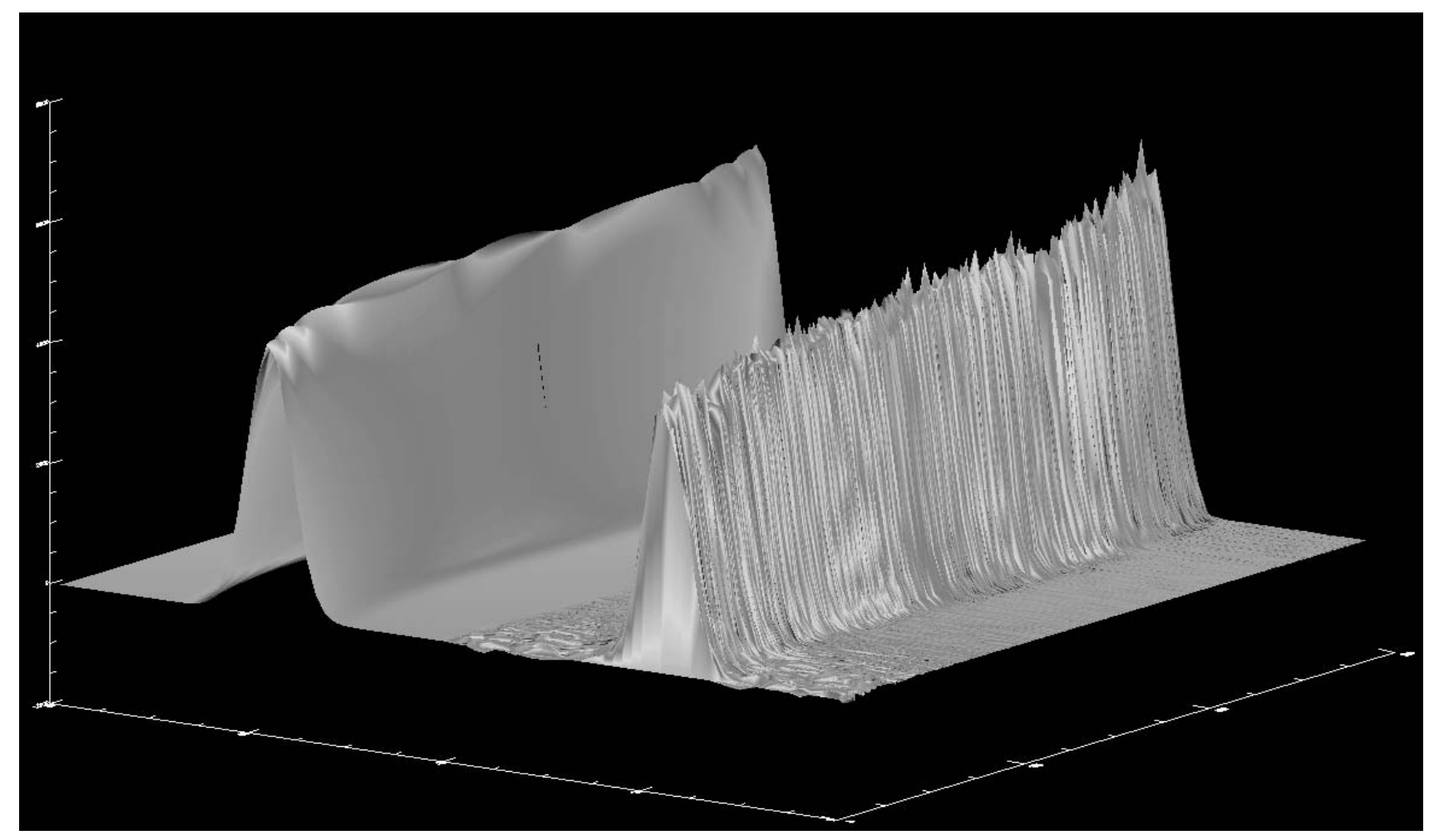

Figure 5. For the pinhole sun spectrum: 3-D Comparison of the real cross-order profile (right) with the one obtained by fitting Gaussian functions (left). The change of the width of the profile along wavelength is clearly visible. 

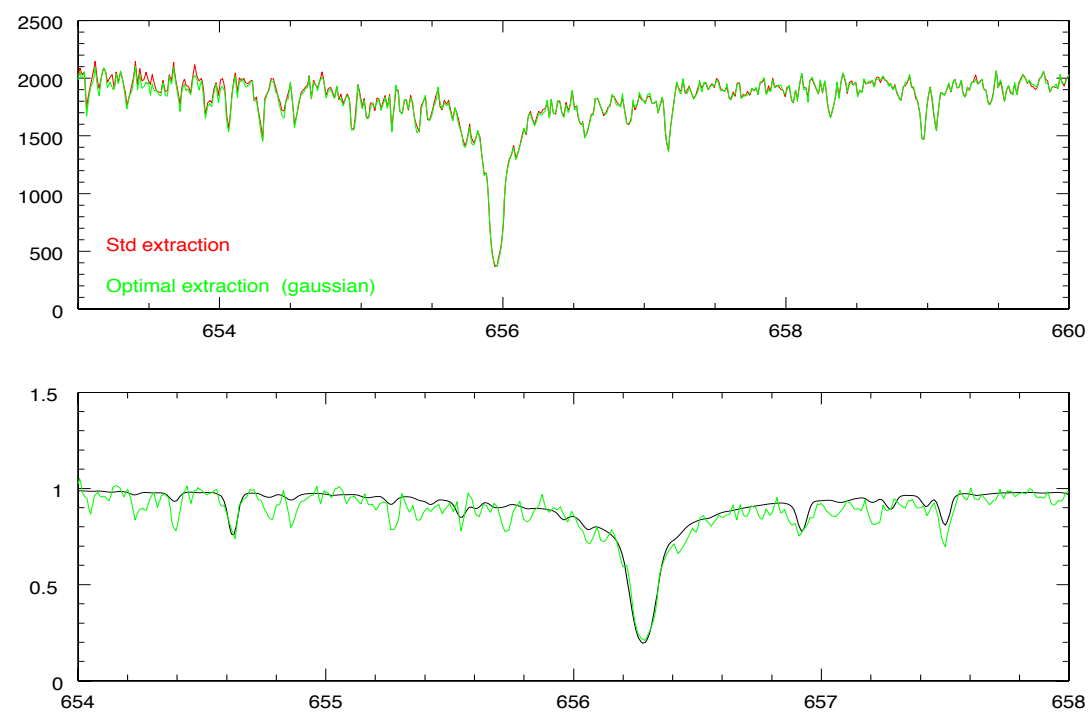

Figure 6. Upper panel: Two Solar spectra extracted from a sun pinhole frame, the green one has been extracted with optimal extraction while the red one has been extracted with standard extraction. The two spctra coincide due to the high S/N and very good "seeing" of the frame. Lower panel: The optimaly extracted solar spectrum is compared to a high resolution solar spectrum degraded to X-shooter resolution. The non fitted absorption features are due to water vapour.

high and the "seeing" is very good (equivalent to 0.5 arcsec).

The lower part of the figure shows the comparison of the extracted spectra with a high quality solar spectrum degraded to the resolution of X-Shooter. The absorption features which are not fitted are due to water vapour trasnsitions.

\subsection{Sky Subtraction in staring mode}

Like any other instrument which operates in the red and near-IR spectral range, correcting for sky background emission is a crucial step in the reduction process. In the case of X-shooter it is complicated by the following:

- The curvature of the orders on the detector.

- The change of plate scale in the direction of the slit.

- Unknown position and shape of the objects in the slit.

The change of plate scale is the unique problem for $\mathrm{X}$-shooter sky subtraction. The change is small, at worst $0.8 \%$. But, as the photon noise on a bright sky emission line can be less than $0.3 \%$, not correcting for the change will lead to systematic errors.

\subsubsection{Super sampling technique}

Super sampling is a recently developed technique for fitting sky background (see the SDSS spectral pipeline or Kelson ${ }^{9}$ ). Its main advantage is that it can be applied to the raw detector format, no resampling is needed, so it is immune to interpolation effects such as change in PSF or noise correlation.

The technique relies on the fact that the spectral lines are tilted, so each pixel samples a slightly different part of wavelength space. An accurate wavelength map is used to re-bin the raw 2D data into a $1 \mathrm{D}$ data set with the wavelength of each pixel as the $\mathrm{x}$-axis and the pixel value in the $\mathrm{y}$-axis. The application of these techniques to 


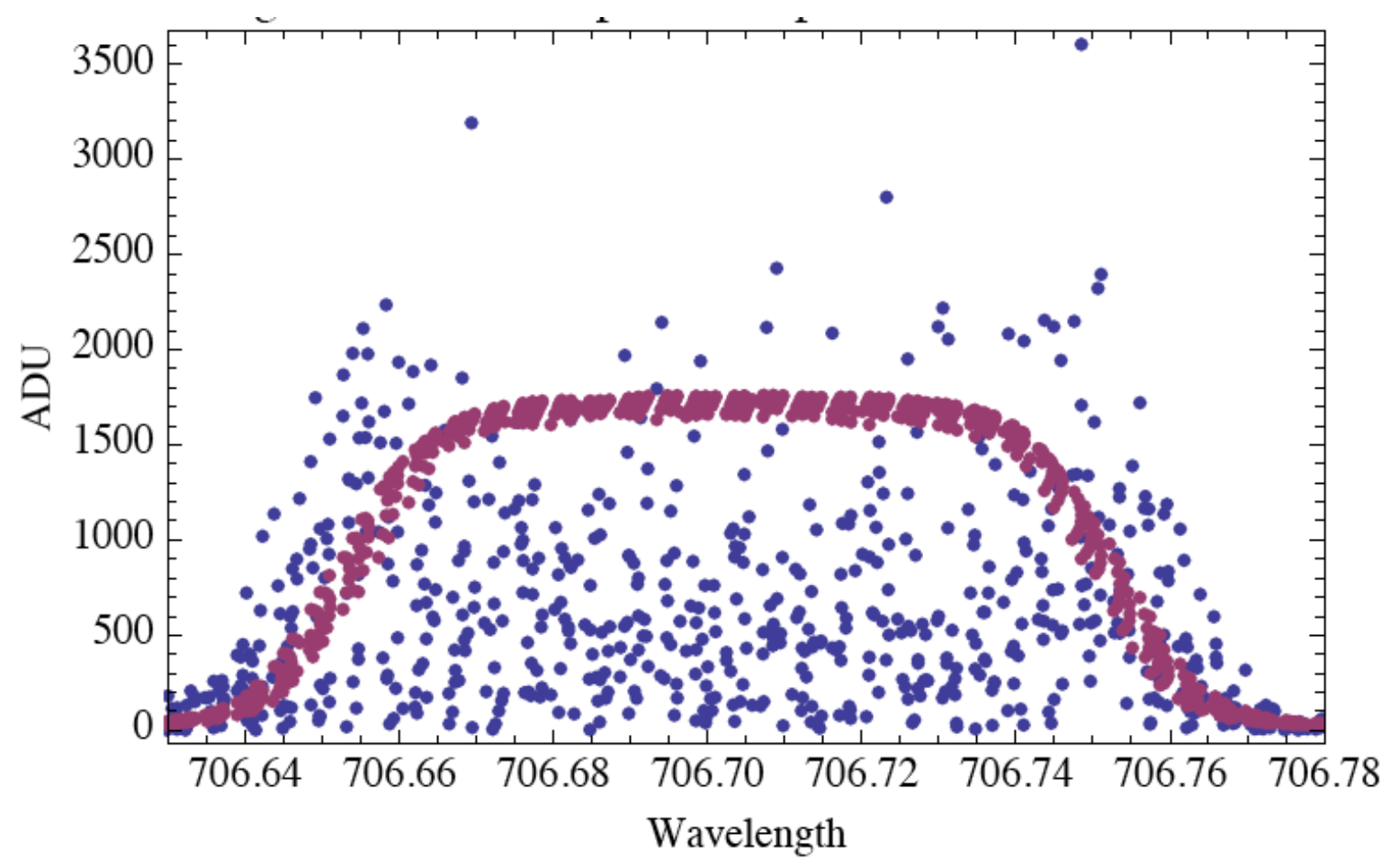

Figure 7. Residuals of the subtraction of a ThAr line from an X-shooter lab frame with our sky model. The actual residuals are in blue while the red line is the $3 \sigma$ contour.

$\mathrm{X}$-shooter data is described in. ${ }^{4}$ We are presently testing these techniques on full slit Th-Ar frames subtracting the lamp lines as if they were sky lines using Mathematica. The results of one of our tests are shown in Fig. 7 where the residuals of the subtraction of the "sky" lines are shown. The maximum flux of the subtracted line being of $\sim 60000$ ADUs, the relative $3 \sigma$ precision is $\sim 2.5 \%$.

\section{FUTURE DEVELOPMENTS}

As shown in this paper, the X-shooter DRS can now perform wavelength calibration on laboratory data, we have started implementing the optimal extraction and sky subtraction algorithm in the code. The version of the code containing these capabilities will be reviewed during Preliminary Acceptance in Europe in Garching in July 2008. Later the DRS will be further developed to handle science observations and will be used during the commissioning observations currently scheduled in September and December 2008. The project should be completed in April 2009.

\section{ACKNOWLEDGMENTS}

We thank all the X-shooter team for their support during this project, in particular P. Bristow for his help during Physical Model Integration. PG, FR, PF, LG and RH thank the Programme Astroparticule for its support.

\section{REFERENCES}

1. S. D'Odorico et al., "X-shooter, a UV to K band, intermediate resolution VLT spectrograph," SPIE Proceedings 6269, p. 98, 2006.

2. S. K. Ramsay, C. M. Mountain, and T. R. Geballe, "Non-thermal emission in the atmosphere above Mauna Kea," MNRAS 259, p. 751, 1992.

3. P. Ballester and M. Rosa, "Modeling echelle spectrograph," APJS 126, p. 563, 1997. 
4. P. Goldoni, F. Royer, P. Francois, M. Horrobin, G. Blanc, J. Vernet, A. Modigliani, and J. Larsen, "Data reduction software of the x-shooter spectrograph," SPIE Proceedings 6269, p. 80, 2006.

5. K. Horne, "An optimal extraction algorithm for ccd spectroscopy," PASP 98, p. 609, June 1986.

6. T. R. Marsh, "The extraction of highly distorted spectra," PASP 101, p. 1032, 1989.

7. N. E. Piskunov and J. A. Valenti, "New algorithms for reducing cross-dispersed échelle spectra," $A \mathscr{E} A \mathbf{3 8 5}$, p. 1095, Apr. 2002.

8. J. F. Donati, M. Semel, B. Carter, D. Rees, and A. Collier Cameron, "Spectropolarimetric observations of active stars," MNRAS 291, p. 658, Nov. 1997.

9. D. D. Kelson, "Optimal techniques in two-dimensional spectroscopy: Background subtraction for the 21st century," PASP 115, p. 688, June 2003. 